\title{
CARACTERÍSTICAS DE LAS SPIN-OFF ACADÉMICAS EN COSTA RICA: UN ESTUDIO EMPÍRICO
}

MAURICIO MONGE AGÜERO

Instituto Tecnológico de Costa Rica mmonge@itcr.ac.cr

\author{
ANTONIO J. BRIONES PEÑALVER \\ Doctorado Europeo en Economía y Gestión de Empresas \\ Universidad Politécnica de Cartagena, España. \\ aj.briones@upct.es \\ DOMINGO GARCÍA PÉREZ DE LEMA \\ Facultad de Ciencias de la Empresa \\ Universidad Politécnica de Cartagena, España. \\ domingo.garcia@upct.es
}

\section{RESUMEN}

El objeto de este trabajo es analizar las características de las spin-off académicas de Costa Rica para contribuir a explicar el proceso de creación de estas y sus características. Contar con información, sobre este tema es de vital importancia para apoyar la toma de decisiones que impulsen su creación. Para ello se ha realizado un estudio empírico a partir de la información de 10 spin-off de Costa Rica. Los resultados muestran que los principales factores que contribuyen para crear una spin-off son poner en práctica sus conocimientos técnicos y la detección de una oportunidad de negocio. Además, el principal problema al que se enfrentan es de índole financiero. Estos resultados son relevantes y de utilidad particularmente a los académicos y a las autoridades universitarias y gubernamentales de cara al fomento de este tipo de organización.

PALABRAS CLAVE: SPIN-OFF ACADÉMICO, CREACIÓN DE EMPRESAS UNIVERSITARIAS, FACTORES DETERMINANTES, CARACTERÍSTICAS

\section{ABSTRACT}

This paper analyzes the characteristics and genesis of academic spin-offs in Costa Rica. Having information on this issue is vital to support the decisions that encourage their creation. This em- pirical study has included the analysis of 10-spin-offs in Costa Rica. The results show that the main factors that contribute to create a spin-off are their abilities to find business opportunities, and that the main problem they face is financial in nature. These results are particularly relevant and useful to academicians, universities and government authorities to promote this type of organization.

KEY WORDS: ACADEMIC SPIN-OFF, UNIVERSITY ENTREPRENEURSHIP, DETERMINANTS, CHARACTERISTICS

\section{INTRODUCCIÓN}

La generación de spin-off universitarias puede contribuir activamente al desarrollo económico de las naciones, apoyando e impulsando los procesos de innovación y desarrollo tecnológico. Por este motivo los gobiernos destinan importantes recursos para su implementación (Lockett, Andy y Wright, 2005b) y el papel de la Universidad empieza a ser visto como un importante conductor del crecimiento económico. De ahí el creciente interés de los académicos por estudiar las spin-off universitarias y se justifica la realización de trabajos que indaguen sobre la comprensión de como las universidades pueden facilitar la creación de nuevas empresas basadas en la investigación (Markman, Gianiodis y Phan, 2008). 
El proceso de evolución de la universidad, concentrada básicamente en la docencia e investigación a una universidad emprendedora, comprometida con el desarrollo económico y las necesidades de su sociedad (Bueno, 2007) tiene sus orígenes en las universidades estadounidenses del Siglo XX, particularmente universidades como el Massachusetts Institute of Technology MIT y la Universidad de Berkeley de los Estados Unidos; las cuales fueron pioneras en la explotación comercial de los resultados de la investigación. El modelo de funcionamiento de estas universidades recibió el apoyo gubernamental, convirtiéndose en un ejemplo para otras universidades, tanto dentro como fuera de los Estados Unidos (Etzkowitz, Henry, Webster, Gebhardt, Cantisano y Branca, 2000). Muchos países, considerando el éxito de este modelo para la generación de riqueza, están comprometidos con las reformas universitarias con miras a incrementar la comercialización de los resultados de la investigación generada con fondos públicos (Wright, Hmieleski, Siegel y Ensley, 2007).

Las spin-off universitarias son empresas desarrolladas por personal académico, que por su naturaleza suelen ser empresas basadas en el conocimiento científico y tecnológico. Estas pueden ser consideradas como el mejor instrumento para la transferencia de la investigación a la sociedad, dado que son innovadoras, de gran aceptación en el mercado y altamente competitivas (ANCES, 2003). La importancia de estudiar la creación del spin-off académico, radica en la capacidad de este instrumento de impactar positivamente en el mercado, al introducir un nuevo producto o servicio de valor agregado. Este valor agregado es resultado de los avances del conocimiento transferidos al mercado como productos y servicios innovadores (Casanova, 2004; OShea, Allen, Morse, O'Gorman, y Roche, 2007; Laborda y Briones, 2010).
Lo anterior constituye un importante reto para cualquier país que pretenda un mejor y mayor desarrollo para sus habitantes. Por ello, consideramos que para Costa Rica las spin-off universitarias constituyen una oportunidad y un reto. El objetivo de este trabajo es analizar las spin-off académicas que surgen en Costa Rica, distinguiendo los factores que afectan al proceso de creación y desarrollo en los primeros años de actividad de la empresa y la valoración del emprendedor académico al crear una spinoff universitaria. Para ello se ha llevado a cabo un estudio empírico, aplicando la metodología basada en el análisis de caso, sobre 10 spin-off académicas. Las preguntas de investigación a responder en este trabajo son fundamentalmente: ¿Qué factores afectan la creación y evolución de las spin-off en Costa Rica?, ¿Cuáles son las características de los fundadores de las empresas?, ¿Cuáles fueron los factores relevantes para la creación de la empresa? y ¿Cuál es la valoración del emprendedor académico respecto de la experiencia como creador de un spin-off? A efectos de comparación, se confrontan los resultados obtenidos en Costa Rica en este trabajo con los resultados obtenidos en España por Ortín et al. (2007).

El trabajo se ha estructurado de la siguiente forma: en primer lugar, se determina el marco teórico, se realiza una revisión de la literatura empírica previa; en segundo lugar, se expone la metodología utilizada y las características de la muestra; en tercer lugar, se lleva a cabo el análisis de los resultados y, finalmente, se exponen las principales conclusiones alcanzadas. 


\section{MARCO TEÓRICO Y REVISIÓN DE ESTUDIOS EMPÍRICOS PREVIOS}

El modelo económico contribuye a incrementar el interés por las actividades emprendedoras, y en particular por aquellas que se desarrollan en las instituciones académicas y centros de investigación. Las universidades gestionan las oficinas de transferencia de tecnología, fomentan las incubadoras de empresas y dotan de financiación los fondos internos de capital/semilla (Rasmussen, Moen y Gulbrandsen, 2006). Por ello, se está dando importancia a la creación de empresas universitarias O'Shea et al. (2004), especialmente Spin-Off Académicas basadas en los resultados de la investigación (Meyer, 2003; Morales, Gutiérrez y Roig, 2009) para incrementar la comercialización de esta (Shane, 2004; Wright et al., 2007).

El proceso de creación de spin-offs académicas tiene sus propias particularidades, debido a que son empresas de alta tecnología y a que surgen dentro de un contexto tradicionalmente no comercial (Vohora, Wright y Lockett, 2004). Este es un campo de estudio reciente en el área del emprendedurismo universitario como instrumento para transferir a la sociedad el conocimiento y los avances generados en las universidades (Autio y Kauranen, 1997; Chiesa y Piccaluga, 2000; OShea et al., 2004; COTEC, 2006; Beraza y Rodríguez, 2010; Rodeiro, Lopez, Gonzalez y Sandias, 2010). Por ello, no podemos desligar el perfil del emprendedor universitario a la actividad socioeconómica que se genera en el proceso de creación de la Spin-Académica. De esta forma, pasamos a describirlo y fundamentar su visión para comercializar los resultados la actividad investigadora.

\section{Emprendedurismo Universitario}

Glassman et al. (2003) definen el "emprendedor académico" como aquel que puede "crear o aprovechar una oportunidad en el contexto universitario"(p.5). Ello implica que comportamientos emprendedores pueden surgir de la implementación de nuevos programas docentes y proyectos investigadores hasta la creación de spin-off académicas; pues incluyen diversos comportamientos derivados de proyectos innovadores, la renovación estratégica de las universidades y los procesos innovadores emprendedores de la institución (Etzkowitz, Henry et al., 2000; Morales, 2008). Louis et al. (1989) hacen mención de cinco grandes tipos de actividades emprendedoras en la universidad: 1) proyectos científicos de gran escala (investigación financiada externamente), 2) obtención de ingresos suplementarios, a través de actividades de consultoría o realización de conferencias, cursos extras, regalías por publicaciones, entre otros, 3) investigación contratada con organizaciones externas, 4) explotación de patentes o secretos industriales y 5) comercialización -participando o formando el capital de empresas basadas en la propia investigación de sus miembros-.

A partir de la anterior clasificación, Jones (1998) elabora una nueva propuesta de actividades emprendedoras, proponiendo los siguientes tipos: (1) Proyectos científicos a gran escala: obteniendo grandes proyectos de investigación financiados externamente, por medio de subvenciones públicas o recursos de fuentes industriales. (2) Investigación contratada: desarrollando proyectos de investigación específicos con el sistema de la universidad para organizaciones externas. (3) Consultoría: la venta de la experiencia del personal científico o tecnológico para resolver un problema específico. (4) Patentes/ licencias: la explotación de patentes o licencias 
de resultados de investigación por parte de la industria. (5) Empresas spin-off: la formación de nuevas empresas u organizaciones para explotar los resultados de la investigación de la universidad. (6) Enseñanza externa: provisión de cursos cortos a personal o estudiantes no universitarios y organizaciones externas. (7) Ventas: venta comercial de productos desarrollados en la propia universidad (por ejemplo, un nuevo software). (8) Servicio de pruebas técnicas: provisión de evaluación y calibración de instrumental o maquinaria para organizaciones o individuos externos a la universidad.

Para Etzkowitz (2003) la creación de grupos de investigación, denominados "quasi-firms", son actividades emprendedoras; ya que el investigador o investigadores deben detectar una oportunidad de mercado en un campo científico, evaluarla, decidir crear una entidad y, posteriormente, obtener los recursos para implementar la idea y gestionarlos por lo que Morales (2008) los incluye dentro de esta definición.

Por tanto, la figura del emprendedor académico ha sido abordada en la literatura, principalmente desde su trayectoria académica, examinando aspectos como el rango académico, la experiencia en actividades emprendedoras dentro de la academia, la calidad y productividad científica y las redes sociales; y muy especialmente en aquellos trabajos que se dirigen hacia el establecimiento de los factores que influyen en la probabilidad de que un investigador decida crear una empresa (Jones-Evans, Dylan 1998; Shane y Khurana, 2003; Shane, 2004; Audretsch, Aldridge y Oettl, 2005; Ding y Stuart, 2006).

La etapa de la carrera profesional en la que se encontraba el emprendedor en el momento de crear su empresa, es una de las características frecuentemente estudiadas. Los modelos económicos del ciclo de vida profesional sugieren que los investigadores crean empresas al final de su carrera (Feldman, Feller, Bercovitz y Burton, 2001; Franzoni y Lissoni, 2006). Una vez alcanzadas sus metas profesionales (estabilidad y premios académicos), los investigadores se plantean obtener retornos financieros de su capital intelectual a través de otro tipo de actividades como, por ejemplo, la creación de empresas spin-off. Para los científicos, crear una empresa es un medio para apropiarse del valor de su propiedad intelectual y para acceder a mecanismos de financiación adicionales para el desarrollo de futuras investigaciones (Feldman et al., 2001). En este mismo sentido, los "modelos del estatus universitario" sostienen que aquellos investigadores que han alcanzado un alto rango académico tienen una mayor probabilidad que otros investigadores de crear empresa (Shane, 2004).

En relación con la experiencia laboral en la academia de los emprendedores, la literatura pone de manifiesto que la investigación científica es la que permite al emprendedor obtener el conocimiento necesario para poder identificar una nueva oportunidad de aplicación comercial (Vohora et al., 2004). Por tal razón, cuanto mayor sea el tiempo de experiencia investigadora, mayor será la probabilidad de descubrir una oportunidad susceptible de explotación a través de la creación de una spin-off. Esta evidencia ha sido aportada por Landry, Amara y Rherrad (2006), quienes encontraron que el número de años de experiencia en investigación influye positivamente en la probabilidad de crear una spin-off.

Otro aspecto frecuentemente analizado, con respecto a los emprendedores académicos, es su calidad científica. Di-Gregorio y Shane (2003) señalan que los científicos de mayor calidad presentarán una mayor probabilidad de crear empresas que los de menor calidad. Por último, Ding et al. (2006) encontraron que los investigadores con mayor probabilidad de crear spin-offs eran aquellos con mayor número de publicacio- 
nes y prestigio. Al igual que el estatus, la eminencia científica de los emprendedores y las instituciones a las que se encuentran vinculados es una señal de la calidad del proyecto de empresa para los inversores.

Di-Gregorio et al. (2003) en un estudio sobre los factores que influyen en que una universidad genere más empresas que otra, hallaron que existe una relación significativa y positiva entre la eminencia de la universidad y el número de empresas creadas por la misma.

Haciendo referencia a la experiencia emprendedora previa en la academia, la literatura relativa al entrepreneurship sugiere que dicha experiencia le permite al emprendedor adquirir las habilidades y capacidades necesarias para la obtención y gestión de los recursos requeridos para llevar a la práctica una idea de negocio (Gartner, 1988). Jones-Evans, Dylan, Steward, Balazs y Todorov (1998) proponen que entre estas actividades emprendedoras se incluyen la obtención de proyectos científicos a gran escala, la investigación contratada, la consultoría, la explotación de patentes a través de licencias, la enseñanza externa, la venta de productos desarrollados en la universidad, el servicio de pruebas técnicas y la creación de spin-offs. Diversos estudios empíricos respaldan la proposición de que los emprendedores académicos acumulan un amplio background emprendedor en la academia. Louis, Blumenthal, Gluck y Stoto (1989) Encontraron que las variables que mejor predecían la participación en el capital de una empresa por parte de un científico eran la existencia de otros comportamientos emprendedores (la consultoría, la obtención de financiación por parte de la industria y las patentes) y el encontrarse en un entorno en el que el entrepreneurship sea la norma.

Shane et al. (2003) hallaron que los inventores que habían licenciado previamente tecnología a otras empresas tenían una mayor probabilidad de crear alguna empresa que los que no habían licenciado; asimismo estos autores sugieren que la experiencia en la obtención de financiación externa aumenta la probabilidad de crear una empresa. Igualmente, Landry et al. (2006) descubrieron que los investigadores que realizaban actividades de consultoría con empresas privadas, agencias gubernamentales u organizaciones asociadas con su campo de investigación muestran una mayor probabilidad de crear una empresa; asimismo, estos autores encontraron evidencia de que, a mayor tamaño del laboratorio en el que trabaje el investigador, mayor será la probabilidad de que cree una spin-off porque dispondrá de un mayor nivel de recursos.

Lo anterior permite deducir que los emprendedores académicos son investigadores que se encuentran en una etapa avanzada de su carrera académica y, por tanto, tienen un alto estatus en su organización de origen, amplia experiencia laboral en la academia, altos niveles de calidad, generalmente los mejores en su área, y un amplio background de experiencia emprendedora en la academia en el momento en que deciden crear sus empresas. Este perfil corresponde a los emprendedores académicos de Estados Unidos y Canadá, dado que la mayoría de trabajos identificados se centran en estos contextos. Adicionalmente, los artículos que hacen referencia a otros países no presentan información en profundidad sobre el perfil de los emprendedores y, en la mayoría de los casos, se orientan hacia la detección de factores que pueden explicar por qué algunos investigadores muestran ciertos comportamientos emprendedores y otros no, lo cual tampoco permite identificar claramente las características del emprendedor; este es el presente caso, en donde no existe un estudio de referencia y este es el primer estudio que se realiza en esta línea y concentrándose la evidencia al caso de las spin-off académicas vinculadas al Instituto Tecnológico de Costa Rica. 


\section{Las spin-off Académicas}

El concepto de spin-off plantea el surgimiento de nuevas actividades económicas creadas a lo interno de una empresa o entidad ya existente que, con su apoyo y supervisión, adquieren independencia y viabilidad, en términos jurídicos, técnicos y comerciales. Las empresas o entidades de las que surgen hacen la función de simiente, sirviendo de apoyo para el despegue del spin-off (ANCES, 2003).

El concepto inicial de spin-off fue el de spin-off industrial (Universia, 2010). En sus orígenes, y dependiendo de la motivación que las genera, se encuentran dos modelos de spin-off. Por una parte está el spin-off reactivo que puede ser de conversión, si su objetivo es gestionar planes explícitos o anticipar soluciones de excedentes de empleo potenciales, o de externalización, cuando se busca preservar una competencia reconocida cuya gestión externa le permite su conversión y desarrollo (CEEl-Valencia, 2009); por otra parte, está el spin-off proactivo, pudiendo ser de tipo estratégico, creado para favorecer el crecimiento externo de actividades o productos en relación directa con la empresa principal; o puede ser de tipo ofensivo, cuando su fin es crear empresas innovadoras organizadas en red, para la explicación de nuevos productos o mercados. Según Rubio (2009) esta clasificación ha sido parcialmente superada, y se habla únicamente de spin-off industrial.

En la definición de la OCDE (2001) (Organización para la Cooperación y el Desarrollo Económico), los spin-offs son: (a) empresas creadas por investigadores del sector público (personal del staff, profesorado o estudiantes); (b) empresas emergentes que disponen de licencias de explotación de tecnologías creadas en el sector público; (c) empresas emergentes sostenidas por una participación directa de fondos públicos, o que fueron creadas a partir de instituciones públicas de investigación. Considerando las anteriores definiciones y desde una amplia perspectiva del término spin-off, este se refiere al proceso de creación de nuevas empresas a partir de otras ya existentes.

Análogamente a los spin-off industriales, surge la modalidad del spin-off académico; las que Pirnay (1998) conceptualiza como un proceso de creación de empresas que cumple con los siguientes requisitos: 1) tiene su origen en una organización existente; se deriva, de una organización madre u organización de origen; 2) involucra a uno o varios individuos, con independencia de cuál sea su estatus o función en la organización de origen; 3) estos individuos (emprendedores académicos) abandonan la organización de origen, para crear una organización nueva, la spin-off.

Conforme el tema ha ido tomando relevancia distintos autores han agregado aspectos importantes que pretenden precisar que se debe entender por spin-off académico. Djokovic y Souitaris (2008), clasifican las definiciones encontradas, en cuanto a tres aspectos fundamentales: 1) resultado del proceso de spin-off universitario. En todas las definiciones, el resultado fue la creación de una empresa. Desde esta perspectiva existe una entidad, de la cual se deriva la otra y se interconectan brevemente a través de un proceso, es decir, la universidad y el spin-off interconectadas través del proceso de transferencia tecnológica, entre las cuales los agentes investigador-emprendedor e inversor, juegan un rol importante como gestores de la nueva empresa (Morales, 2008), 2) las partes implicadas: a) la organización de origen, de la que se obtiene la tecnología que se transfiere. En el caso del spin-off universitario, la organización origen es la universidad u otro centro de investigación 
(Roberts y Malonet, 1996); b) el creador de la tecnología, que es la persona que la desarrolla, de modo que esta pasa de ser un resultado de investigación básica a un elemento susceptible de comercialización; c) el empresario, que tiene por objetivo crear una empresa nueva centrada en la explotación de la tecnología y el inversor, que proporciona la financiación para la nueva empresa; y 3) los elementos que se transfieren en el proceso, que pueden ser la tecnología y/o las personas. La transferencia de la tecnología es un elemento necesario del proceso de creación de spin-off académico por lo que se pueden encontrar diferencias en cuanto a qué se considera tecnología y en cuanto a la necesidad o no de la transferencia de personas.

Las definiciones sobre spin-off académico que se centran en cuanto a qué se considera tecnología, se distinguen dos grupos de definiciones.

1) Las que consideran que la tecnología debe estar protegida formalmente, a través de una patente. En este caso, la spin-off universitaria es una empresa creada para explotar un elemento de propiedad intelectual que tiene su origen en una universidad o centro de investigación (McQueen y Wallmark, 1982; Di-Gregorio et al., 2003; Lockett y Wright, 2005); 2) Están las definiciones que consideran tecnología a cualquier conocimiento producido en la universidad sin que tenga que estar necesariamente formalizado (Pirnay, Bernad Surlemont y Nlemvo, 2003).

En cuanto a la transferencia de personas en el proceso de spin-off, se encuentran dos tipos de definiciones (Smilor, Dietrich y Gibson, 1993): 1) las que consideran que la transferencia de personas es una condición necesaria para una spinoff universitaria; y 2) las que consideran que la transferencia de personas no es una condición necesaria para una spin-off universitaria.
Para Van Dierdonck y Debackere (1988) utilizan los términos extrapreneurial spin-offs e intrapreneurial spin-offs para caracterizar los casos en los que el inventor deja la universidad y en los que se queda, y Nicolau y Birley (2003) diferencian entre spin-offs tecnológicas, ortodoxas e híbridas. La primera categoría de spin-offs tecnológicas incluye a las spin-offs en las que únicamente se transfiere la tecnología; las spin-offs ortodoxas implican, además, la transferencia del empresario, que abandona la universidad. En cambio, cuando las spin-offs son híbridas, el empresario mantiene su puesto en la universidad y lo compatibiliza con su actividad en la spin-off (Nicolau et al., 2003).

Pinary (2003) divide las spin-off académicas desde dos perspectivas 1) el estatus del individuo en la organización, las spin-off se dividen en estudiantiles y académicas. Las spin-offs estudiantiles son aquellas creadas por miembros de la comunidad estudiantil, con poco bagaje investigador; mientras que las académicas son las empresas creadas por un individuo de una comunidad "científica", incluyendo personas con experiencia investigadora, como es el caso de los profesores, ayudantes, investigadores y estudiantes de doctorado; y 2) La naturaleza del conocimiento transferido desde la universidad a) Spin-off universitarias dedicada a explotación comercial de conocimiento codificado b) Spinoff universitarias dedicadas a la explotación comercial de conocimiento tácito. Otro elemento que tiende a caracterizar a las spin-off académicas, según Steffensen et al.,(1999) es que estas pueden surgir de una forma espontánea o planeada desde la universidad, es decir si cuentan o no con el apoyo explícito de la universidad. El término spin-off académico es reciente, ello implica que no existe un consenso en bibliografía sobre su definición, lo que dificulta el análisis 
(Morales, 2008; Aceytuno y Cáceres, 2009). Por lo que será necesario proponer una definición que incluya los aspectos más relevantes tratados en las diferentes definiciones estudiadas.

\section{METODOLOGÍA DE LA INVESTIGACIÓN}

En este informe se identifica el proceso de creación de las spin-off académicas de Costa Rica y sus características, lo que permitirá establecer los factores que tienen mayor incidencia en su creación, todo ello con la finalidad de que los tomadores de decisiones cuenten con elementos objetivos que les guíen, a fin de promover la creación de este importante tipo de empresas. La metodología escogida en esta investigación para analizar las variables que la literatura menciona como relevantes respecto del proceso de creación de las spin-off académicas, es el método del caso, apoyándose en la investigación descriptiva, porque se pretende identificar los elementos clave o variables que inciden en un fenómeno; y exploratoria porque es un primer acercamiento de las teorías, métodos e ideas del investigador a la realidad objeto de estudio (Maxwell, 1998), como es el caso del presente trabajo, el cual pretende responder a los siguientes interrogantes: ¿Existen o no spin-off académicas surgidas del quehacer de la Universidad en Costa Rica? De existir, ¿qué tipo de spin-off son las que se han creado?, ¿cuáles son sus características más relevantes?, ¿qué factores afectaron la creación y evolución de las empresas?, ¿cuáles son las características de los fundadores de las empresas?, ¿cuáles fueron los factores relevantes o motivantes en la creación de la empresa? y ¿cuál es la valoración del emprendedor académico respecto de la experiencia como creador de un spin-off?
Como medio de comparación o Pattern Matching (Yin, 2009) se utilizó el estudio empírico: La creación de spin-off universitarias en España: Características, determinantes y resultados (Ortín, Salas, Trujillo y Vendrell, 2007). Esta investigación aportó los datos de las características de las spin-off académicas y el perfil del emprendedor académico. La investigación se circunscribe a empresas de Costa Rica fundadas -en consonancia con la misión de la institución- por profesores e investigadores durante el periodo que estuvieran en ejercicio de sus funciones docentes, o que ya las hayan finalizado y sin importar si el conocimiento es tácito o explícito.

La población objeto de la presente investigación está compuesta por 10 spin-off académicas. Se identificaron 12 emprendimientos; se entrevistó a 10 emprendedores, ya que a dos no fue posible localizarlos. Las entrevistas se realizaron entre el 1 de octubre de 2009 y el 10 de febrero de 2010, en forma personal. En la tabla 1 se caracterizan de forma descriptiva las spin-off que configuran la muestra. En esta tabla se asignará un 1 (uno) si la empresa cumple con el requisito y un 0 (cero) si esta no lo cumple. De esta tabla se puede apreciar que las características generales de la muestra son: las spin-off son empresas derivadas de la actividad universitaria; están vinculadas al área de ciencia y tecnología; fueron fundadas por docentes e investigadores y surgieron de forma espontánea impulsadas por la iniciativa del emprendedor académico ante una oportunidad de negocio; comercializan tanto conocimiento tácito como explícito; el 50\% de los emprendedores abandonó la universidad (ortodoxa); el 40\% se mantiene vinculado (hibrida); y un 10\% transfirió el conocimiento a través de una patente para su comercialización (tecnológica). 


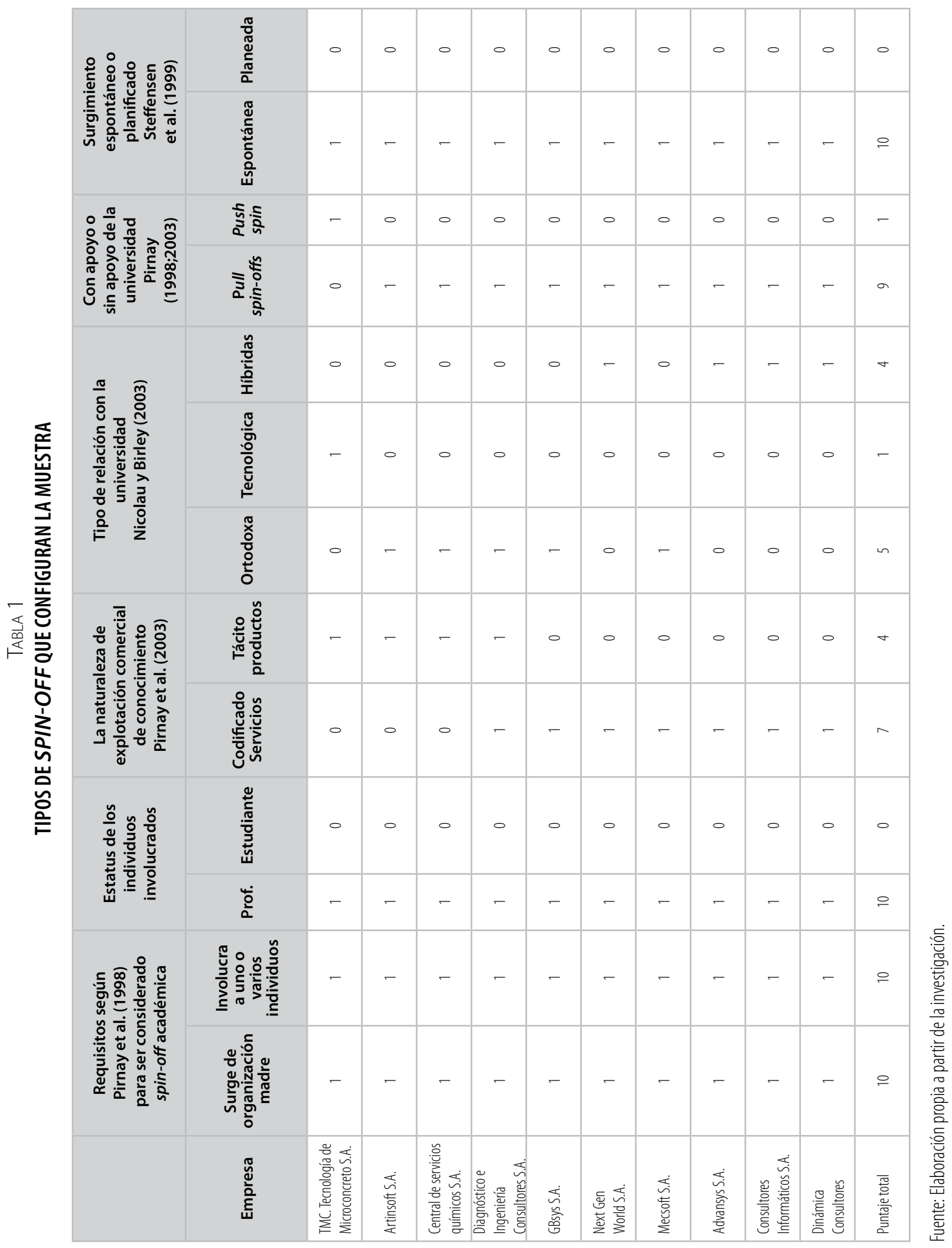




\section{ANÁLISIS DE RESULTADOS}

\section{Características generales}

de las spin-off académicas

A continuación se exponen las características generales de las spin-off académicas analizadas. En primer lugar, consideramos el sector al que pertenece la empresa y el porcentaje destinado a la Investigación y Desarrollo (I+D) sobre las ventas. En la tabla 2 comprobamos cómo el 70\% de los emprendimientos está concentrado en el área de las tecnologías de la información; y el 30\% restante existe una spin-off en cada sector: química/forestal, metalurgia y construcción. Estos resultados son similares a los obtenidos en España donde el 54\% pertenecen al sector de tecnología de la información o el 70\% en Estados Unidos (Ortín et al., 2007). Respecto al porcentaje destinado de I+D sobre las ventas, los resultados muestran que en promedio, las empresas destinan un $14,67 \%$ de sus ventas al presupuesto de I+D. El máximo valor destinado al presupuesto de I+D fue de un 30\% y el mínimo de un 5\%. Lo que en contraste con las spin-off académicas españolas invierten en promedio un 36,8 \%, es decir, invierten un 2,5 veces más, no obstante al no contar con datos absolutos de las ventas brutas no se puede establecer una comparación dado también las diferencias del tamaño en empleado y en antigüedad de las spin-off de Costa Rica.

En la tabla 3 se aprecian diferencias entre las spin-off en España y Costa Rica. En España las spin-off son más jóvenes que las encontradas en Costa Rica, dado que el 75\% de las spin-off espa- ñolas tienen menos de cinco años mientras que el promedio de las spin-off de Costa Rica tiene 17,42 años. La distribución que presenta es de un 30\% para las que se encuentran entre cinco y 10 años, un 40\% entre 11 y 20 años, un 20\% entre 21 y 25 años y, finalmente, un 10\% entre 26 y 30 años. El tamaño medio también resulta diferente, mientras en Costa Rica tienen de promedio 22 empleados, el tamaño en España es de 8,34 empleados. El 100\% de las empresas en Costa Rica está bajo la figura de sociedad anónima, mientras que el 89,7\% de las empresas españolas son sociedades de responsabilidad limitada.

En cuanto al capital fundacional, el $46,2 \%$ de las spin-off españolas se fundaron con un capital de 5000€ (equivalente a US\$ 6855,00 en el momento de realizar la investigación) (Ortín et al., 2007), mientras que en Costa Rica el 70\% de las spinoff se fundaron con un capital social que osciló entre los US\$2000 y US\$3000, lo que es coincidente en el sentido de que fueron fundadas con un bajo capital y prácticamente salvando el requisito del capital mínimo que exige la ley para crear una empresa. Respecto a la procedencia del capital social, el 100\% de las spin-offen Costa Rica menciona que sus socios y ellos aportaron el capital para fundar la empresa. De los que contestaron, el 75\% aportó de sus propios ahorros y el 25\% de una combinación de ahorros, en contraste con las spin-off españolas donde el 90\% fue capital de los fundadores.

TABLA 2

\section{SECTOR Y \% DESTINADO A LA I+D COMO PARTE DE LAS VENTA}

\begin{tabular}{|l|c|l|c|}
\hline \multicolumn{1}{|c|}{ Sector } & \% & \multicolumn{1}{|c|}{ Inversión anual (I+D) } & \% \\
\hline Tecnología de información & 70 & Máximo & $30 \%$ \\
\hline Química/Forestal & 10 & Mínimo & $5 \%$ \\
\hline Metalurgia & 10 & Promedio & $14,67 \%$ \\
\hline Construcción & 10 & Mediana & $14,66 \%$ \\
\hline
\end{tabular}

Fuente: Elaboración propia a partir de las entrevistas. 
TABLA 3

\section{CARACTERÍSTICAS GENERALES DE LAS SPIN-OF}

\begin{tabular}{|c|c|c|}
\hline & Spin-off en Costa Rica & Spin-off en España \\
\hline \multirow{4}{*}{ Años de operación de la empresa } & $\begin{array}{c}\text { Años } \\
30 \% \text { entre } 5 \text { y } 10\end{array}$ & \multirow{4}{*}{$\begin{array}{l}75 \% \\
\text { menos de } 5 \text { años }\end{array}$} \\
\hline & $40 \%$ entre 11 y 20 & \\
\hline & $20 \%$ entre 21 y 25 & \\
\hline & $10 \%$ entre 26 y 30 & \\
\hline Número medio de empleados & 22 & 8,34 \\
\hline$\%$ sociedades de responsabilidad limitada & 0 & $89,7 \%$ \\
\hline \% sociedades anónimas & 100 & n.d. \\
\hline \% de capital social con que se funda la empresa & $\begin{array}{l}\text { US\$ } 2000 \text { y } 3000 \\
70\end{array}$ & $\begin{array}{l}\text { US\$ } 6855(<5000 €) \\
46\end{array}$ \\
\hline$\%$ de capital aportado por los fundadores & 100 & 90 \\
\hline \% de empresas con ayudas públicas a la financiación & 10 & 37,7 \\
\hline
\end{tabular}

Fuente: Elaboración propia a partir de las entrevistas y de Ortín A. et al. (2007).

En las tablas 4 y 5 se presentan algunas de las características de los empleados de las spin-off académicas de Costa Rica, lamentablemente no se cuenta con información para contrastar los resultados. La spin-off académica con más empleados tiene 88 y la de menos empleados cuenta con 2. El total de empleo generado por las spin-off analizadas asciende a 205 puestos de trabajo de los cuales el 77,07\% son hombres y un 22,93\% son mujeres. Esto quiere decir que en estas empresas de ciencia y tecnología existe una relación de 3,4 hombres por mujer.

En la tabla 5 se muestra dónde se concentra la mayor cantidad de empleados. Estos se concen- tran en el área de producción (71,22\%), seguida del área de investigación con un 17,07\% y, por último, un 11,71\% en el área administrativa. Adicionalmente, se observa que a nivel administrativo la participación de hombres y mujeres es 1 a 1; no obstante, en el área de producción la superioridad numérica de hombres es evidente, de tal manera que hay 2,40 hombres por mujer; en el área de investigación es aún más marcada la diferencia, que llega a 4,83 hombres por mujer. Esto evidencia la desigualdad de género existente en las empresas spin-off, las cuales tienen fundamento en ciencia y tecnología según la investigación realizada por Queralt (2008). 
TABLA 4

TOTAL DE EMPLEADOS Y GÉNERO EN LAS SPIN-OFF DE COSTA RICA

\begin{tabular}{|l|c|c|c|}
\hline \multicolumn{1}{|c|}{ Empresa } & Total de empleados & Hombres & Mujeres \\
\hline Total de empleados & 205 & $77,07 \%$ & $22,93 \%$ \\
\hline Promedio & 22 & 15,8 & 6,2 \\
\hline Máximo & 88 & 53 & 35 \\
\hline Mínimo & 2 & 1 & 0 \\
\hline
\end{tabular}

Fuente: Elaboración propia a partir de las entrevistas y de Ortín et al. (2007).

TABLA 5

DISTRIBUCIÓN DE EMPLEADOS POR ÁREA FUNCIONAL Y GÉNERO

\begin{tabular}{|l|c|c|c|}
\hline & Administración & Producción & Investigación \\
\hline Empleados por área & $11,71 \%$ & $71,22 \%$ & $17,07 \%$ \\
\hline Hombres & $50,00 \%$ & $70,55 \%$ & $82,86 \%$ \\
\hline Mujeres & $50,00 \%$ & $29,45 \%$ & $17,14 \%$ \\
\hline
\end{tabular}

Fuente: Elaboración propia a partir de las entrevistas y de Ortín et al. (2007).

Factores relevantes y principales

problemas en la creación de la empresa

En la tabla 6 se muestran los resultados comparativos con España. Los dos factores más relevantes para los académicos que crearon una spin-off en Costa Rica fueron, primero "poner en práctica sus conocimientos técnicos" y segundo "la detección de una oportunidad de negocio".
En España esta situación es similar aunque se invierten las posiciones. Contrariamente, el factor menos importante para los académicos en Costa Rica, y que fue designado en último lugar, fue "afán de ganar más dinero que trabajando a sueldo". Esto difiere ligeramente respecto a los académicos españoles, que lo sitúan en tercer lugar. En el caso de España el factor menos relevante es el impulso de la empresa o centro donde trabajaba.

TABLA 6

\section{FACTORES RELEVANTES EN LA CREACIÓN DE SPIN-OFF ACADÉMICA}

\begin{tabular}{|l|c|c|}
\hline \multicolumn{1}{|c|}{ Ítem } & COSTA RICA & ESPANA \\
\hline Poner en práctica conocimientos técnicos & 1 & 2 \\
\hline Detección de una oportunidad de negocio & 2 & 1 \\
\hline Lo propuso o animó la empresa o instituto donde trabajaba & 3 & 6 \\
\hline Prestigio o estatus del empresario & 3 & 4 \\
\hline Ventajas de trabajar por cuenta propia & 3 & 5 \\
\hline Afán de ganar más dinero que trabajando a sueldo & 4 & 3 \\
\hline
\end{tabular}

Fuente: Elaboración propia a partir de la investigación y de Ortín, et al. (2007). 
Como se observa en la tabla 7, los emprendedores académicos manifiestan haber tenido principalmente problemas financieros, lo cual es también consecuente con las investigaciones de Hurst y Lusardi (2003) En este sentido Ortin et al., (2007), señalan que existen abundantes investigaciones en las cuales se pone en evidencia la relación entre la propensión a crear una empresa y la riqueza del emprendedor. En el 23\% de las respuestas de los entrevistados, se menciona que el principal problema al que se enfrentó fue no contar con recursos financieros suficientes para desarrollar su emprendimiento. Esta variable es la que presenta un mayor peso; posteriormente un $14 \%$ menciona la carencia de equipo y maquinaria; un 12\% la tecnología inmadura; la carencia de recursos humanos capacitados y los problemas de corte técnico un 12\%; luego, con un menor porcentaje se mencionaron como problemas la comunicación y la ubicación geográfica; y, por último, el 15\% señaló otros problemas.

TABLA 7

\section{PRINCIPAL PROBLEMA AL QUE SE ENFRENTÓ AL CREAR LA EMPRESA}

\begin{tabular}{|l|c|c|}
\hline & $\begin{array}{c}\text { Número de } \\
\text { Respuestas }\end{array}$ & $\%$ \\
\hline Financiero & 8 & $23,53 \%$ \\
\hline $\begin{array}{l}\text { Carencia de } \\
\text { maquinaria }\end{array}$ & 5 & $14,71 \%$ \\
\hline Técnicos & 4 & $11,76 \%$ \\
\hline $\begin{array}{l}\text { Recurso humano } \\
\text { capacitado }\end{array}$ & 4 & $11,76 \%$ \\
\hline Tecnología inmadura & 4 & $11,76 \%$ \\
\hline Gestión empresarial & 2 & $5,88 \%$ \\
\hline Comunicación & 2 & $5,88 \%$ \\
\hline Ubicación geográfica & 2 & $5,88 \%$ \\
\hline Mercado inmaduro & 1 & $2,94 \%$ \\
\hline $\begin{array}{l}\text { Desorden } \\
\text { administrativo }\end{array}$ & 1 & $2,94 \%$ \\
\hline El producto & 1 & $2,94 \%$ \\
\hline Total de respuestas & 34 & $100,00 \%$ \\
\hline
\end{tabular}

Fuente: Elaboración propia a partir de las entrevistas.

\section{Factores relevantes en los primeros años de actividad}

\section{Acceso a recursos financieros}

En las spin-off de Costa Rica manifestaron no haber recibido ayuda económica, ni por la universidad, ni por el Sistema Bancario Nacional. Esto tiene su justificación porque la universidad no está facultada para ello y la banca de desarrollo es de creación reciente y a la fecha aún no existen empresas que brinden capital de riesgo. En contraste, el 40\% de las spin-off españolas sí han recibido apoyo financiero, aunque consideran el proceso de acceso a dichas ayudas muy complejo (Ortin et al., 2007).

\section{Asesoramiento en la creación y gestión de la empresa}

Las spin-off académicas en Costa Rica no recibieron asesoramiento ni capacitación durante la creación y gestión de la empresa, mientras que en el caso de las empresas españolas cerca del 90\% contaron con esta clase de apoyo (Ortin et al., 2007).

\section{Experiencia profesional}

Contar con experiencia en gestión de empresas o de tecnología, según Ortin et al. (2007) y Clarysse y Moray (2004), es algo especialmente positivo para crear una spin-off. No obstante, en el caso de los emprendedores académicos de Costa Rica solo un 20\% tenía experiencia en gestión empresarial. Esto viene a señalar que, aunque la experiencia profesional previa es deseable, no resulta una condición única para la creación de empresas y su éxito posterior. 


\section{Valoración personal de la experiencia}

En general, se puede decir que los emprendedores académicos entrevistados en Costa Rica presentan un alto grado de satisfacción por haber tomado la decisión de emprender su proyecto productivo, dado que el 90\% mencionó sentirse orgulloso de haberlo logrado y el 80\% menciona que la experiencia ha valido la pena en cuando a desarrollo personal. Un 60\% menciona como otro aspecto por el cual sentirse orgulloso del emprendimiento, que "la experiencia ha permitido mejorar la situación económica". En comparación con los emprendedores españoles se puede señalar que estos también manifiestan una alta valoración de la experiencia emprendedora desde la perspectiva del desarrollo personal. Aunque Ortin et al. (2007) mencionan que no están tan satisfechos en lo que se refiere a mejorar su nivel de renta económica.

\section{CONCLUSIONES}

En la sociedad actual, donde se afirma que la competitividad de las empresas y su salud económica depende de su capacidad de incorporar tecnología (Beraza et al., 2010), basado en el conocimiento científico, estudiamos la spin-off académica, que surge de un sistema de innovación que vincula a la ciencia con el desarrollo socioeconómico y está constituido por tres actores básicos: la universidad y/o centro de investigación, las empresas y el gobierno (Etzkowitz, Henry y Leydesdorff, 2000).

Esta investigación hace una contribución al describir las características de las spin-off académicas en Costa Rica y los factores determinantes del proceso de creación. Se trata de un primer esfuerzo en ese sentido y la conclusión general que se extrae de este estudio es que falta todavía mucho conocimiento que permita una comprensión suficiente de un fenómeno tan importante.

Los métodos cualitativos utilizan técnicas de obtención de información no estructurada, flexible y de tipo psicológico y sociológico, por ello se utilizó la metodología de caso. Sus técnicas trabajan con muestras reducidas de las que se obtiene abundante conocimiento. Evidentemente sus resultados no son cuantificables ni extrapolables al conjunto de una población. No obstante, su utilidad radica en su potencia para la descripción de hechos y en cómo consigue explicar sus motivaciones con datos (Martínez, 2006).

Los hallazgos de esta investigación ponen de manifiesto que las empresas entrevistadas son spin-off académicas, derivadas de la actividad universitaria; están vinculadas al área de ciencia y tecnología; fueron fundadas por docentes e investigadores y surgieron de forma espontánea impulsadas por la iniciativa del emprendedor académico; comercializan tanto conocimiento tácito como explícito; el 50\% se clasifica como ortodoxa porque el emprendedor abandonó la universidad; el 40\% se consideran híbridas porque el emprendedor se mantiene vinculado a su universidad; y un 10\% son tecnológicas, porque transfirió el conocimiento a través de una patente para su comercialización. Sobre las características generales de las spin-off académicas se concluye que: las spin-off académicas tienen en promedio 17,42 años de estar en funcionamiento, el 70\% se encuentra en el área de tecnología de la información; el 40\% tiene menos de 20 años; en promedio tienen 22 empleados; y existe un predominio del sexo masculino, se fundaron con un capital entre $1400 €$ y $2200 €$ y no recibieron apoyo económico del gobierno para fundar la empresa.

El ambiente en que estas empresas surgieron fue difícil dado que el $80 \%$ de los emprende- 
dores no tenía experiencia previa en gestión de empresas al iniciar su emprendimiento y no contaron con apoyo o estímulo institucional. No obstante, el 90\% mencionó sentirse orgulloso de haber logrado crear la spin-off y el $80 \%$ que la experiencia ha valido la pena en cuando a su desarrollo personal.

Estos resultados son relevantes y de utilidad. Este trabajo es útil para los académicos a fin de conocer el proceso de creación de una spin-offy los factores relevantes y problemas a los que se va a enfrentar. Para las autoridades académicas a fin estimular la creación de spin-off en sus instituciones, favoreciendo el asesoramiento y la utilización de sus instalaciones. Finalmente, es de utilidad para las autoridades gubernamentales a fin de mejorar el entorno financiero y jurídico que permita un mayor desarrollo de spin-off en su tejido productivo como un factor determinante del crecimiento económico de su país.

Este estudio presenta varias limitaciones que sugieren vías de investigación futuras. En primer lugar, este trabajo se circunscribe al ámbito de una universidad pública, el Instituto Tecnológico de Costa Rica, y fue realizado con una muestra muy limitada. En futuros estudios sería oportuno analizar un contexto geográfico más amplio como el de Centro América, al objeto de conocer sus problemas y fortalezas de modo que las conclusiones obtenidas sirvan de motor de crecimiento de estos países. Además, es un estudio exploratorio y descriptivo, por lo que, en futuras investigaciones, aportes estadísticos más profundos y la consideración del impacto de variables como la innovación y el tipo de cultura implantado en las spin-off sobre su rendimiento, permitiría profundizar en los factores de éxito de este tipo de organizaciones.

\section{Referencias}

Aceytuno, M., y Cáceres, R. (2009). Elementos para elaboración de un marco de análisis para el fenómeno de las spin offs universitarias. Revista de Economía Mundial(23), 23-52.

ANCES. (2003). La Creación de Empresas de Base Tecnológica. Una experiencia práctica Proyecto para la promoción de empresas innovadoras de base tecnológica. Valencia, España: ANCES, CEIN y otros.

Audretsch, A., Aldridge, T., y Oettl, A. (2005). The knowledge filter and Economic Growth: The role of Scientist Entrepreneurship. Discussion Paper on Entrepreneurship, Growth and Public Policy, 1106, 66.

Autio, E., y Kauranen, I. (1997). Technologist-entrepreneurs versus non-entrepreneurial technologists: analysis of motivational triggering factors. Entrepreneurship y Regional Development, 6, 315-328.

Beraza, J. M., y Rodríguez, A. (2010). Factores determinantes de la utilización de las spin-off como mecanísmo de transferencia de conocimiento en las universidades. Investigaciones Europeas de Dirección y Economía de la Empresa, 16 (2), 115-135.

Bueno, E. (2007). La Tercera Misión de la Universidad: El reto de la transferencia del conocimiento. Revista de Investigación en Gestión de la Innovación y Tecnología,(41).

Casanova, J. (2004). La experiencia de los CEEIS Españoles apoyando EIBTS. ANCES, 619-626.

CEEI-Valencia. (2009). SPIN-OFF, la fuerza centrífuga de las ideas Retrieved 07-07, 2010, from http://www.ceeivalencia.com/index.php?op=8yn=826

COTEC. (2006). Tecnología e innovación en España. [Informe]. Informe COTEC 2006.

Chiesa, V., y Piccaluga, A. (2000). Exploitation and diffusion of public research: the case of academia spin-off companies in Italy. RyD Management, 30(4), 329-339.

Di-Gregorio, D., y Shane, S. (2003). Why do Some Universities Generate More Start-ups than Others? Research Policy , 32, 209-227. 
Ding, W., y Stuart, T. (2006). When Do Scientists Become Entrepreneurs? The Social Structural Antecedents of Commercial Activity in the Academic Life Sciences. American Journal of Sociology, 112(1), 97-144.

Djokovic, D., y Souitaris, V. (2008). Spinouts from academic institutions: a literature review with suggestions for further research. The Journal of Technology Transfer, 33(3), 225-247. doi: 10.1007/s10961-006-9000-4

Etzkowitz, H. (2003). Research groups as [']quasi-firms': the invention of the entrepreneurial university. [doi: DOI: 10.1016/S0048-7333(02)00009-4]. Research Policy, $32(1), 109-121$.

Etzkowitz, H., y Leydesdorff, L. (2000). The dynamics of innovation: from National Systems and "Mode 2" to a Triple Helix of university-industry-government relations. Research Policy, 29(2), 109-123. doi: 10.1016/ S0048-7333(99)00055-4

Etzkowitz, H., Webster, A., Gebhardt, C., Cantisano, T., y Branca, R. (2000). The future of the university and the university of the future: evolution of ivory tower to entrepreneurial paradigm. [doi: DOI: 10.1016/S00487333(99)00069-4]. Research Policy, 29(2), 313-330.

Feldman, M., Feller, I., Bercovitz, J., y Burton, R. (2001). Understanding Evolving University-Industry Relationshipspp. . In M. L. In: Feldman, A. (Ed.), Innovation Policy in the Knowledge-based Economy, (pp. 171-188). United States of America.: Kluwer Academic Publishers.

Franzoni, C., y Lissoni, F. (2006). Academic entrepreneurship, patents and spin-offs: critical issues and lessons for Europe. Paper presented at the Centro di Ricerca sui Processi di Innovazione e Internazionalizzazione CESPRI, Università Commerciale "Luigi Bocconi".

Gartner, W. B. (1988). Who Is an Entrepreneur?" Is the Wrong Question. American Journal of Small Business, 12(4), 11-32.

Glassman, A., Moore, R., Rossy, G., Neupert, K., Napier, N., Jones, D., y Harvey, M. (2003). Academic entrepreneurship: Views on balancing the Acropolis and the Agora. Journal of Management Inquiry "1 12( 4), 353374.

Hurst, E., y Lusardi, A. (2003). Liquidity Constraints, Household Wealth, and Entrepreneurship. Journal of Political Economy, Vol. 112, pp. 319-47, April 2004.
Jones-Evans, D. (1998). Universities, technology transfer and spin-off activities-academia entreneurship in different European Regions (U. O. GLAMORGAN, Trans.). In T. S.-E. R. PROJECT (Ed.), Targeted Socio-economic Research Project.

Jones-Evans, D., Steward, F., Balazs, K., y Todorov, K. (1998). Public sector entrepreneurship in Central and Eastern Europe: A study of academic spin-offs in Bulgaria and Hungary. Journal of Applied Management Studies, 7(1), 59-59-76.

Laborda, F., y Briones, A. (2010). Innovación y Tecnología Factores de dirección estratégica de los agronegocios en Costa Rica y la Región de Murcia (pp. 99-116). Costa Rica-España: Universidad Politécnica de Cartagena.

Landry, R., Amara, N., y Rherrad, I. (2006). Why are some university researchers more likely to create spin-offs than others? Evidence from Canadian universities. [doi: DOI: 10.1016/j.respol.2006.09.020]. Research Policy, 35(10), 1599-1615.

Lockett, A., y Wright, M. (2005). Resources, Capabilities, Risk Capital and the Creation of University Spin-out Companies. Research Policy, 34, 1043-1057.

Lockett, A., y Wright, M. (2005 b). Resources, Capabilities, Risk Capital and the Creation of University Spin-out Companies. Research Policy „, 34, 1043-1057.

Louis, K., Blumenthal, D., M.Gluck, y Stoto, M. (1989). Entrepreneurs in Academe: Behaviours among Life Scientist. Administrative Science Quarterly, 34(1), 110-131.

Louis, K. S., Blumenthal, D., Gluck, M., y Stoto, M. (1989). Entrepreneurs in Academe: Behaviours among Life Scientist. Administrative Science Quarterly, 34(1), 110131.

Markman, G. D., Gianiodis, P. T., y Phan, P. (2008). Full-Time Faculty or Part-Time Entrepreneurs. IEE Transactions on Engineering Management, 55(1), 29-36.

Martínez, P. C. (2006). El método de estudio de caso estrategia metodológica de la investigación científica. pensamiento y gestión,, 20, 165-193.

McQueen, D., y Wallmark, T. (1982). Spin-off Companies from Chalmers University of Technology Technovation,, 1 301-315. 
Meyer, M. (2003). Academic Entrepreneurs or Entrepreneurial Academics? Research-based Ventures and Public Support Mechanisms. RyD Management, 33(2), 107-115.

Morales, S. T. (2008). El Emprendedor académico y la decisión de crear spin off: Un análisis del caso Español. Tesis Doctoral, Universitat de Valencia, Valencia.

Morales, S. T., Gutiérrez, A., y Roig, S. (2009). The entrepreneurial motivation in academia: a multidemensional construct. [Working Paper Series 2009-07]. [Las motivaciones del emprendedor académico: Construcción multidimensional]. Ingenio (CSIC-UPV), 2008/11.

Nicolau, N., y Birley, S. (2003). Academia Networks in a Trichotomous Categorisation of University Spinouts. Journal of Business Venturing "1 18, 333-359.

OCDE. (2001). Introduction: The New Spin on Spin-offs Retrieved 07-07, 2010, from http://www.oecd.org/ document/57/0,3343, en_2649_34409_2046201_1_ 1_1_1,00.html

Ortín A., P., Salas, V., Trujillo, M. V., y Vendrell, F. (2007). La creación de Spin-off universitarios en España: Características, determinantes y resultados. 32.

Ortín, P., Salas, V., Trujillo, M. V., y Vendrell, F. (2007). La creación de Spin-off universitarios en España: Características, determinantes y resultados. 32.

OShea, R., Allen, T., Morse, K., O'Gorman, C., y Roche, F. (2007). Delineating the anatomy of an entrepreneurial university: the Massachusetts Institute of Technology experience. [Delineando la anatomì de un emprendedor universitario: La experiencia del Instituto Tecnológico de Massachusetts]. RyD Management, $37(1), 16$.

OShea, R., Allen, T., Morse, K., O'Gorman, C., Roche, F., y Cáceres, R. (2004). Universities and Technology Transfer: A review of academic entrepreneurship literature. A Review of academic Entrepreneurship Literature". Irish Journal of Management, 25 (2), 11-29. .

Pirnay, F. (1998). Spin off et essaimage: de quoi sàgit-il? Une reveu de la littératuré. Colloque International Francophone sur la PME, Metz-Nancy, 22-24 October.
Pirnay, F., Bernad Surlemont, y Nlemvo, F. (2003). Toward a Typology of University Spin-offs Small Business Economics (Vol. 21, pp. 355-369). Netherlands: Kluwer Academic Publishers.

Queralt, L. (2008). Trayectorias Laborales y Personales de Mujeres profesionales de carreras tradicionalmente masculinas: El caso de las egresadas del Instituto Tecnológico de Costa Rica. Máster en Género y Políticas de Igualdad Máster en Género y Políticas de Igualdad, UNIVERSIDAD DE VALENCIA, Valencia, España.

Rasmussen, E., Moen, Ø., y Gulbrandsen, M. (2006). Initiatives to promote commercialization of university knowledge. [doi: DOI: 10.1016/j.technovation.2004.11.005]. Technovation, 26(4), 518-533.

Roberts, E. B., y Malonet, D. E. (1996). Policies and structures for spinning off new companies from research and development organizations. RyD Management, $26(1), 17-48$.

Rodeiro, D., S. Lopez, L. Gonzalez, y A. Sandias. (2010). Capital structure determinants of university spin-offs. [Article]. Academia-Revista Latinoamericana De Administracion(44), 65-98.

Rubio, A. (2009). Spin-off, la fuerza centrífuga de las ideas Centros europeros de empresas innovadoras (CEEI-Valencia). Retrieved 27-06-2010, 2010, from http://www. ceei-valencia.com/index.php?op $=8 y n=826$

Shane, S. (2004). Academic Entrepreneurship University spin offs and Wealth Creation. Londres: Edward Elgar Publishing.

Shane, S., y Khurana, R. (2003). Bringing individuals back in: the effects of career experience on new firm founding. Industrial and Corporate Change, 12(3), 519-543.

Smilor, R., Dietrich, G., y Gibson, D. (1993). The entrepreneurial University: the role of higher education in the United States in technology commercialization and economic development. International Social Science Journal,, 45(1), 1-11.

Steffensen, M., Rogers, E. M., y Speakman, K. (1999). Executive Forum: Spin-offs From Research Centers at a Research UniverUniversia. (2010). Spin-off: una nueva forma de emprender, una forma de investigar Retrieved 27-06-2010, 2010, from http://investigacion.universia.es/spin-off/index.htm 
Van-Dierdonck, R., y Debackere, K. (1988). Academic Entrepreneurship at Belgian Universities. RyD Management, 18(4), 341-353.

Vohora, A., Wright, M., y Lockett, A. (2004). Critical junctures in the development of university high-tech spinout companies. Research Policy, 33(1), 147-175. doi: 10.1016/S0048-7333(03)00107-0

Wright, M., Hmieleski, K. M., Siegel, D. S., y Ensley, M. D. (2007). The role of human capital in technological entrepreneurship. Entrepreneurship: Theory and Practice, 31(6), 791-806. doi: 10.1111/j.1540-6520.2007.00202.x

Yin, R. (2009). Discovering the future of the case study method in evaluation research. Evaluation Practice, 15(3), 283-290. doi: Doi: 10.1016/0886-1633(94)90023-x

Recibido: 21-11-2011

Aceptado: 15-02-2012 